\title{
Analysis of the Helical Milling Technology and the Vibration Characteristics
}

\author{
Kangwei Chen ${ }^{1, a}$, Xi Wang ${ }^{2, b}$ and Yafei Yao ${ }^{2, c}$ \\ School of Xiamen University, Xiamen 361000, China;
}

\begin{abstract}
Keywords: Spiral milling hole; Difficult-to-machine materials; Wavelet transform; Vibration signal Abstract. With the improvement of aircraft assembly digitized and new aviation difficult-to-machine materials are widely used, conventional drilling is more and more cannot satisfy the needs of processing. Spiral milling hole technology as a new machining technology is relatively traditional drilling especially difficult machining titanium alloy materials and composite materials shows many advantages, has been applied to foreign aircraft assembly site successfully, but bright took strict foreign technology blockade. This article is based on the principle of spiral milling hole using wavelet analysis method for the spiral milling hole with traditional drilling vibration signals were analyzed.
\end{abstract}

\section{The spiral milling hole technical characteristics}

Hole processing technology is rising in recent years, a new type of hole process, especially for new aviation difficult-to-machine materials hole processing aspect shows good prospects for development. Tool movement in the process of spiral milling hole formed by superposition of three kinds of sports: around its own axis rotation movement of cutting tool and cutting tool machining hole axis orbit around the axial feed and the cutting tool. This kind of special form of movement determines the spiral milling hole technology has many great advantages in machining [1] :

1 , spiral milling hole is eccentric processing, using a tool can work out a series of different diameter hole, greatly reduce the types of cutting tools and tool change frequency, improve efficiency, reduce the cost. 2 , under the same processing conditions compared with traditional drilling method, spiral milling drilling hole axial cutting force is a 1/10-1/8 [2], which can greatly reduce the stratification in the composite system of hole, and can effectively improve the processing automation. 3 , heat dissipation fully spiral milling machining process, greatly reduce the incidence of surface burn in the processing, at the same time effectively reduce tool wear. 4, eccentric processing make clearance space fully, smooth chip removal. Reduced as a result of cutting tool wear and cutting force increases; And reduce the adverse impact of the cutting quality of hole wall.

\section{The research status of spiral milling hole}

Spiral milling hole technology many advantages make it become the hotspot in the present study, the principle of spiral milling hole had a large number of basic research at home and abroad, and in the screw hole milling equipment development, processing technology parameters optimization.

Spiral milling technology abroad started early, has been in aircraft manufacturing companies such as Boeing and airbus, SAAB is applied to aircraft assembly process. Mechanism and actual application feasibility in spiral milling holes, Henrik Kihlman [3] and found in the process of spiral milling hole axial force is about $1 / 8$ to $1 / 10$ that of the conventional drilling, verified the feasibility of industrial robot automation spiral milling hole. In spiral milling hole processing technology, R.I yer [4], the screw hole milling technology in the application of hard to machining tool steel material AISI D2, eliminating the reaming process, improve the processing efficiency; In the face of foreign technology blockade, domestic universities and scientific research units had a lot of research work. Qin Xu of tianjin university and others in the spiral milling hole end executor of independent research and development has made breakthrough progress, spiral milling process of hole cutting force model is established, through the genetic algorithm optimization of cutting parameters and tool Angle [5]. Analysis of nanjing university of aeronautics and astronautics li liang spiral milling dynamics, 
aluminum alloy under the large feed hole processing quality, surface roughness of robot helical milling hole modeling is studied [6];

\section{The spiral milling hole movement form}

Spiral milling hole drilling process is different from the traditional process, cutting tool's rotation and axial feed at the same time also revolves around the axis of the processing hole, the cutting tool along the spiral feeding in rotation at the same time.

This kind of movement determines the spiral milling machining process of the particularity of tool cutting work, simultaneously side blade milling way machining and drilling side blade in a similar way. Main parameters in the process of spiral milling machining has four: tools around its own axis rotation speed, around the hole axis of revolution speed, cutting tool along the axial feed speed, eccentricity, in the reasonable selection of parameters in actual processing of the final processing quality will have great influence on [7] - [8].

\section{Test equipment and test design}

1 , The processing platform

VMC650E type vertical machining center adopts the vertical frame layout, pillar fixed on the bed, headstock (Z), move up and down along the column slide along the longitudinal mobile bed (Y), table along the slide transverse direction $(\mathrm{X})$ structure. The main technical parameters is as follows:

Main motor power: $5.5 \mathrm{~kW} / 7.5 \mathrm{~kW}$. The spindle speed: $50 \mathrm{r} / \mathrm{min}$ to $8000 \mathrm{r} / \mathrm{min}$; Feed speed: 1 $\mathrm{mm} / \mathrm{min}$ to $10000 \mathrm{~mm} / \mathrm{min}$; Table size (length * width) : $900 \mathrm{mmx} 420 \mathrm{~mm}$; Linear axis stroke (X/Y/Z) : $650 \mathrm{~mm} / 400 \mathrm{~mm} / 500 \mathrm{~mm}$.

2,Machining

Tensile aluminum alloy 7050 - T7451 aerospace. 7050 is a high strength heat treatable alloys, high strength and resistance to spalling corrosion and stress corrosion cracking resistance performance. Which often used in aircraft structures free forging and die forging parts.

3 , The signal acquisition system

Vibration sensor, the model for the CA - YD - 1182.

\section{The traditional test system of drilling hole}

The traditional system of drilling hole is now commonly used form a system of hole processing, application and aperture equal bit drilling of vertical direction of material processing.

\section{Test equipment}

Shank drill, drill shank $10 \mathrm{~mm}$, bit $18 \mathrm{~mm}$ in diameter, high speed steel material.

\section{Test design}

According to the characteristics of the traditional system of drilling hole processing, will test the speed of the bit and the longitudinal feed speed is set to change. Bit is $18 \mathrm{~mm}$ in diameter, pretensioning in aviation aluminum alloy 7050 - T7451 material processing several aperture to $18 \mathrm{~mm}$, $6 \mathrm{~mm}$ deep blind hole, the test parameters for drilling speed and longitudinal feed speed, detailed parameters and schemes as shown in table 1 .

Table 1 The traditional system of drilling hole parameters

\begin{tabular}{ccc}
\hline Group number & Bit speed(r/min) & Longitudinal feed speed $(\mathrm{mm} / \mathrm{min})$ \\
\hline 1 & 800 & 80 \\
2 & 800 & 120 \\
3 & 800 & 160 \\
4 & 1200 & 80 \\
5 & 1200 & 120 \\
6 & 1200 & 160 \\
7 & 1600 & 80 \\
8 & 1600 & 120 \\
9 & 1600 & 160 \\
\hline
\end{tabular}




\section{The test of spiral milling hole}

\section{Test equipment}

1. The tool

Tool is TPA50 degrees vertical milling cutter, tungsten steel material, the parameters of $10.0 * 4$ $* 25 * 10 \mathrm{~d} * 751 \mathrm{t}$.

\section{Rainfall distribution on 10-12 test design}

To explore the influence of three elements of the helical milling machining, design multiple sets of parameters for different process parameters under the condition of spiral milling hole test, test process parameters including tool rotation speed, traverse speed and axial feeding speed for, detailed parameters and schemes as shown in table 2 .

Table 2 Spiral milling hole parameters

\begin{tabular}{|c|c|c|c|}
\hline Group number & Rotation speed(r/min) & Transverse feed speed $(\mathrm{mm} / \mathrm{min})$ & Longitudinal feed speed $(\mathrm{mm} / \mathrm{r})$ \\
\hline 1 & 1500 & 320 & 0.2 \\
\hline 2 & 1500 & 320 & 0.5 \\
\hline 3 & 1500 & 320 & 0.8 \\
\hline 4 & 1500 & 640 & 0.2 \\
\hline 5 & 1500 & 640 & 0.5 \\
\hline 6 & 1500 & 640 & 0.8 \\
\hline 7 & 1500 & 960 & 0.2 \\
\hline 8 & 1500 & 960 & 0.5 \\
\hline 9 & 1500 & 960 & 0.8 \\
\hline 10 & 2160 & 320 & 0.2 \\
\hline 11 & 2160 & 320 & 0.5 \\
\hline 12 & 2160 & 320 & 0.8 \\
\hline 13 & 2160 & 640 & 0.2 \\
\hline 14 & 2160 & 640 & 0.5 \\
\hline 15 & 2160 & 640 & 0.8 \\
\hline 16 & 2160 & 960 & 0.2 \\
\hline 17 & 2160 & 960 & 0.5 \\
\hline 18 & 2160 & 960 & 0.8 \\
\hline 19 & 3000 & 320 & 0.2 \\
\hline 20 & 3000 & 320 & 0.5 \\
\hline 21 & 3000 & 320 & 0.8 \\
\hline 22 & 3000 & 640 & 0.2 \\
\hline 23 & 3000 & 640 & 0.5 \\
\hline 24 & 3000 & 640 & 0.8 \\
\hline 25 & 3000 & 960 & 0.2 \\
\hline 26 & 3000 & 960 & 0.5 \\
\hline 27 & 3000 & 960 & 0.8 \\
\hline
\end{tabular}




\section{Transformation processing vibration signal}

\section{The wavelet transform}

Wavelet transform is a kind of new transformation analysis method, inherited and developed the localization of the short time Fourier transform (STFT), at the same time overcome the shortcomings, such as the window size is not on frequency change. Its main characteristic is through the transform can fully highlight some aspects of the problem characteristics, can in time (space) frequency localization analysis, through the telescopic translation operations (functions) of signal gradually multi-scale refinement, ultimately achieve high frequency time segment, the low frequency in the frequency segment, can automatically adapt to the requirement of time-frequency signal analysis, which focuses on the arbitrary signal details, solved the difficult problem of Fourier transform, become the Fourier transform has been a great breakthrough on the scientific method of [9].

\section{Improve wavelet}

Two-dimensional discrete wavelet transform is one of the most effective method of using Mallat algorithm computational complexity is high, high to storage space requirement, for hardware implementation. Improve algorithm is a more efficient and effective implementation method of the wavelet transform, known as the second generation wavelet transform. Daubechies has proved that any discrete wavelet or two order filtering transformation with finite length filters can be decomposed into a series of simple improvement steps, so can use Mallat algorithm of wavelet, can use to improve algorithm implementation [10].

\section{The wavelet transform result data analysis}

In traditional drilling experiment was carried out respectively and spiral milling machining after test, the collected data import lifting wavelet transform Matlab procedures, to make simple changes and find a better separation layer. After processing will be separated and reconstructing the data in the form of ASCII is stored as a text file again. In the number of processed data, write a program to test data selectively import, within the same chart box present different processing parameters of the data processing image at the same time. By contrast, relevant conclusions.

\section{Traditional drilling vibration signal analysis}

First, select 2, 5, 8 sets of data images for comparison, as shown in figure 1 .

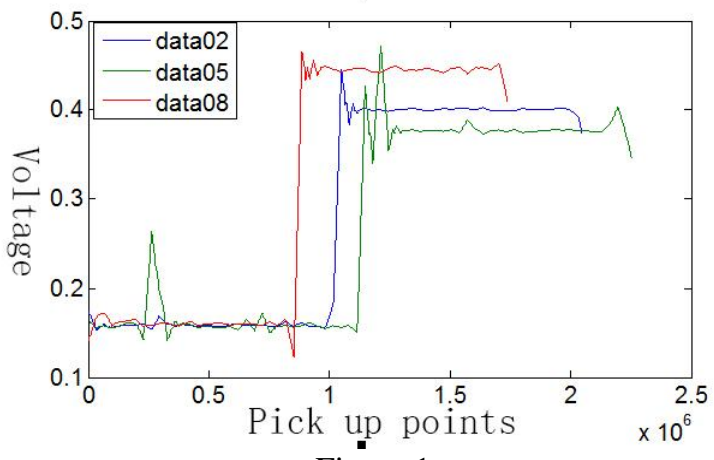

Figure 1

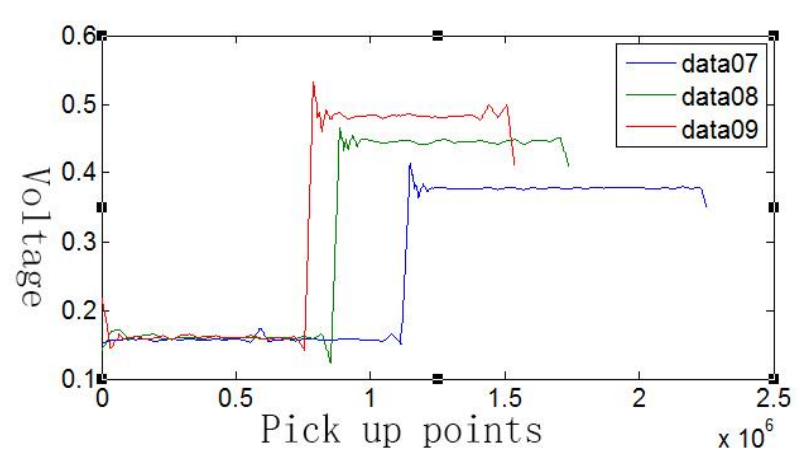

Figure 2

2, 5, 8 groups of the same process parameters for the feed speed of $120 \mathrm{~mm} / \mathrm{min}$. Tool rotation speed of $800 \mathrm{r} / \mathrm{min}$, respectively, $1200 \mathrm{r} / \mathrm{min}$ and $1600 \mathrm{r} / \mathrm{min}$, form contrast. And in the original vibration signal after lifting wavelet transform, the image contrast, 8 group voltage higher than 5 groups, group 5 voltage higher than that in group 2 voltage. Group are 5 vibration obviously, 8 and 5 groups of vibration significantly in the second group. Also can both control group according to the characteristics, can be speculated that in conventional drilling, the feed rate unchanged, increases with the increase of tool rotation speed and vibration, but the impact is not big.

Second, select 7, 8, 9 sets of data for comparison, as shown in figure 2. 7, 8, 9 groups of the same process parameters for cutting tool rotation speed of $1600 \mathrm{r} / \mathrm{min}$. The feed rate is respectively 80 $\mathrm{mm} / \mathrm{min}, 120 \mathrm{~mm} / \mathrm{min}, 160 \mathrm{~mm} / \mathrm{min}$. And in the original vibration signal after lifting wavelet transform, the group of voltage higher than group $8,9.8$ voltage higher than 7 group voltage. The 
groups vibration is 8,9 and 8 groups are 7 vibration is obvious. Also can both control group according to the characteristics, presumably in traditional drilling, keep the tool rotation speed is constant, increases with the increase of feed speed and vibration, but the impact is not big.

\section{spiral milling machining vibration signal analysis}

First, select 5, 14, 23 sets of data for comparison, as shown in figure 3. 5, 14 and 23 group of the same set of process parameters of $640 \mathrm{~mm} / \mathrm{min}$ and transverse feed speed of $0.5 \mathrm{~mm} / \mathrm{r}$ longitudinal feed speed. Tool rotation speed $1500 \mathrm{r} / \mathrm{min}$, respectively $2160 \mathrm{r} / \mathrm{min}$ and $3000 \mathrm{r} / \mathrm{min}$, form contrast. And in the original vibration signal after lifting wavelet transform, the image contrast, 23 voltage higher than 14 group voltage, 14 voltage higher than 5 group voltage. 23 are 14 vibration group obviously, and 14 group are 5 vibration is obvious. The equivalent properties of the control group all can show the characteristics of speculate on spiral milling machining, keep the transverse longitudinal feed speed is constant, increases with the increase of tool rotation speed and vibration.

Second, 11, 14, 17 sets of data for comparison, as shown in figure 4.

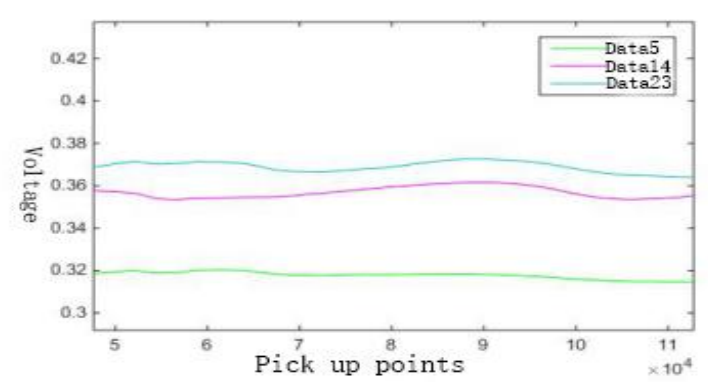

Figure 3

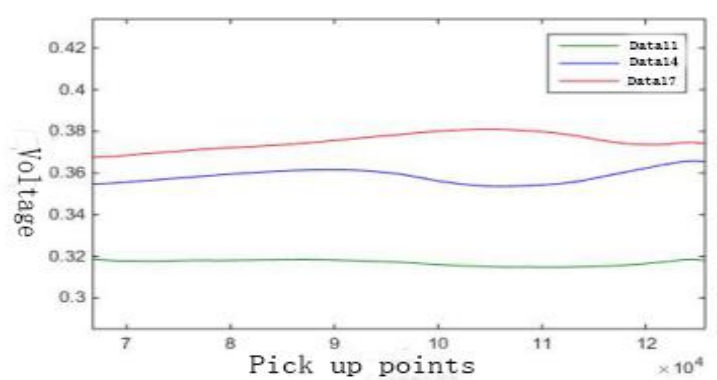

Figure 4

11,14 and 17 groups of the same process parameters of $2160 \mathrm{r} / \mathrm{min}$ of tool rotation speed and longitudinal feed velocity of $0.5 \mathrm{~mm} / \mathrm{r}$. The traverse speed, respectively for $320 \mathrm{~mm} / \mathrm{min}, 640$ $\mathrm{mm} / \mathrm{min}, 960 \mathrm{~mm} / \mathrm{min}$, form contrast. And in the original vibration signal after lifting wavelet transform, the image contrast, the voltage is higher than 14 groups voltage, 17 and 14 voltage higher than 11 group voltage. Groups are 14 vibration obviously, 17 and 14 groups are 13 vibration is obvious. The rest of the equivalent properties of the control group all can show the characteristics of can speculate on spiral milling machining, the tool rotation speed and longitudinal feed rate unchanged, with transverse vibration feed rate increases.

\section{the comparison of two kinds of vibration signal processing way}

To maintain the same cutting speed compare the parameters, selection of traditional drilling group of experiment data image with spiral pores $5 \sim$ image as a comparison group test data, as shown in figure 5.

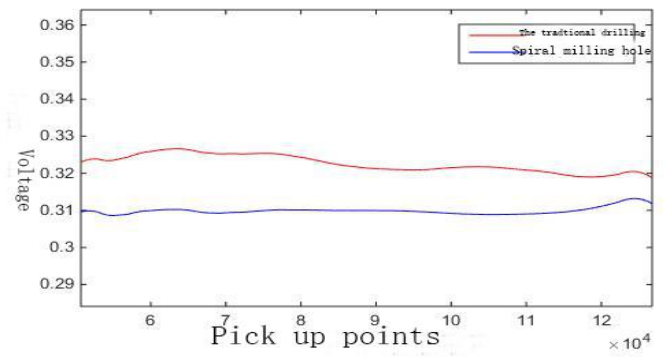

Figure 5

In the cutting speed phase at the same time, the spiral milling hole test voltage is lower than the traditional drilling test voltage, the spiral milling machining vibration than traditional drilling small vibration. In this perspective, screw milling hole processing technology is compared with traditional drilling technology is more stable and better. 


\section{Summary}

Air through the pre-draft aluminum alloy 7050 - T7451 adopts the traditional process method and the spiral milling drilling the hole process method to test and control of process parameters under different methods set, get the following conclusion:

In conventional drilling, the feed rate unchanged, increases with the increase of tool rotation speed and vibration, but little impact; And keep the tool rotation speed is constant, increases with the increase of feed speed and vibration, but little impact; In spiral milling machining, transverse longitudinal feed and feed speed is constant, increases with the increase of tool rotation speed and vibration; And the tool rotation speed and longitudinal feed rate unchanged, with transverse vibration feeding speed increases; And cutting tool rotation speed and the transverse feed speed is constant, with the longitudinal vibration of feed speed increases; In keep cutting speed and the same normal work, the vibration of the spiral milling machining than traditional drilling small vibration, more stable and better processing effect can be thought.

\section{References}

[1] Yafei Wang, spiral milling hole cutting tools of aviation refractory materials research, zhejiang university, 2014.

[2] Herik Kihlman, Ingvar Eriksson, Mark Ennis. Robotic Oribital Drilling of Structures for Aerospace Applications. SAE Aerospace Automated Fastening Conference and Exhibition, 2002

[3]Henrik Kihlman, Ingvar Eriksson,Mark Ennis . Robotic Orbital Drilling of Structures for Aerospace Applications. SAE Aerospace Automated Fastening Conference and Exhibition, 2002

[4] R.I yer, P.K oshy, E.N we elical milling: an enabling technology for hard machining precision holes in AISI D2 tool steel. The International Journal of Machine Tools\&Manufacture 47 (2007) 205-2007

[5]Xuda Qin, Shimao Chen, Weicheng Liu, etc. The spiral milling technology in the manufacturing and assembly of the aviation industry development application [J]. Aviation manufacturing technology, 2009 (6) : 58-60.

[6] Shan Y C, He N, Li L, et al., spacex milling hole of aerospace of al Alloy with big pitch, the Transactions of Tianjin University, 2011, 17:329-329

[7] Wang Gongsong. Difficult-to-machine materials of spiral milling hole technology research [D]. Dalian university of technology, 2012.

[8] Linjing Gui. Based on the intractability of MQL machining material spiral milling technology research [D]. Tianjin university, 2012.

[9] Defeng Zhang. MATLAB wavelet analysis [M]. Mechanical industry publishing house, 2009. [10] Zhou Wei. MATLAB wavelet analysis, a senior technology [M]. Xi 'an university of electronic science and technology press, 2006. 